\title{
Dielectric dispersion and piezoelectric resonance in benzil single crystals grown by Bridgman-Stockbarger technique
}

\author{
M V SHANKAR and K B R VARMA* \\ Materials Research Centre. Indian Institute of Science. Bangalore 560012. India \\ MS received 26 June 1996; revised 29 July 1996
}

\begin{abstract}
Large single crystals of an organic nonlinear optical (NLO) material. benzil, have been grown by employing an indigenously built Bridgman-Stockbarger crystal growth system. The dielectric constant $\left(\varepsilon_{\mathrm{r}}\right)$, dielectric loss tangent $(\tan \delta)$ and impedance $(Z)$ of these crystals, monitored along the crystallographic a-axis, as a function of frequency in the $100 \mathrm{kHz}-10 \mathrm{MHz}$ range, exhibit sharp resonance effects in the $100-300 \mathrm{kHz}$ region. These strong resonance effects are attributed to the piezoelectric nature of the crystal. The origin of the appearance of the sharp peaks in the frequency variation of $\varepsilon_{r}$ and $\tan \delta$ is in response to the elastic resonances induced via the externally applied electric field. The resonance peak positions shift towards lower frequencies with increase in temperature as a consequence of the decrease in the stiffness coefficient $\left(C_{11}\right)$ of the crystal.
\end{abstract}

Keywords. Benzil: dielectric dispersion: piezoelectric resonance.

\section{Introduction}

Recent interest in the nonlinear optical (NLO) properties of organic crystals has motivated the quest for understanding the underlying basic polar properties that are responsible for the observed NLO responses. Studies on the frequency response of the dielectric constant of these solids give an insight into the rate of the polarization processes that occur in these systems and also help one to explore the possibility of characterizing these materials for resonance enhanced electro-optic properties (Bosshard et al 1993). Benzil is a well known organic NLO crystal (Jerphagnon 1971) and crystallizes in the trigonal space group: P3 $2_{1}$ (Brown and Sadanaga 1965), at room temperature. In this communication. we report the details with regard to the growth of benzil single crystals by Bridgman-Stockbarger technique and the frequency dependence of their dielectric properties. The results are discussed in light of the piezoelectric nature of these crystals.

\section{Experimental}

Large single crystals of benzil (length: $35 \mathrm{~mm}$, dia: $12 \mathrm{~mm}$ ) were grown by employing an indigenously built Bridgman-Stockbarger (BS) crystal growth system, which was specially designed for growing single crystals of low-melting organic NLO materials (Shankar et al 1994). The crystal growth system essentially consisted of a vertical two-zone furnace, an ampoule lowering mechanism based on planetary gear system and two proportional-type temperature controllers $\left( \pm 0.5^{\circ} \mathrm{C}\right.$ accuracy). The temperature of the melting zone and the growth zone were maintained at $110^{\circ} \mathrm{C}$ and $70^{\circ} \mathrm{C}$. respectively. Ampoules made of glass were used for the growth of these crystals and an ampoule iowering rate of $\approx 1.3 \mathrm{~mm} / \mathrm{h}$ was employed. The schematic of the BS system that was used to grow the crystals for the present study is shown in figure 1 . The purity 


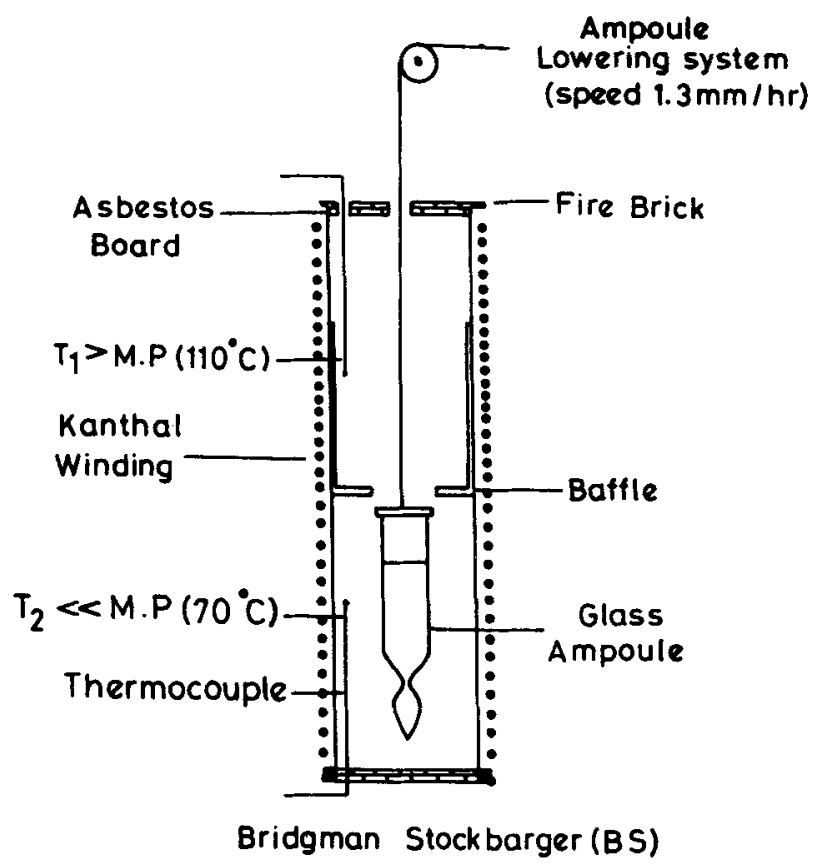

Figure 1. Schematic of the Bridgman-Stockbarger crystal growth system.

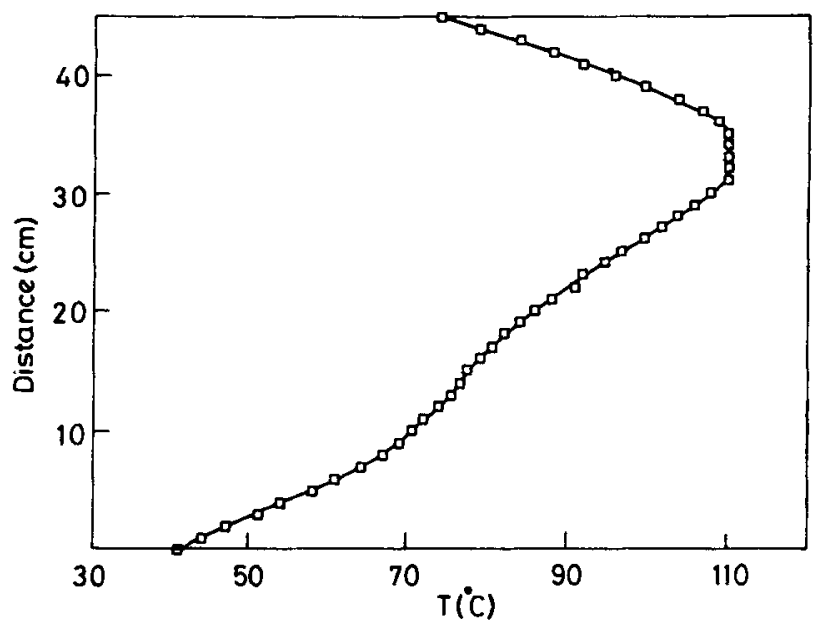

Figure 2. Temperature profile of the BS furnace, used for the growth of benzil single crystal.

of the starting material is a key prerequisite for growing good quality crystals by BS method. Commercially obtained benzil was purified by repeated recrystallization from acetone and by zone refining. The melting point of the starting material was confirmed by subjecting it to differential scanning calorimetric (DSC) studies.

The dielectric measurements on silver electroded, oriented rectangular crystal plates, of dimensions $7.3 \times 6.15 \times 0.75 \mathrm{~mm}^{3}$ were carried out, as a function of frequency 


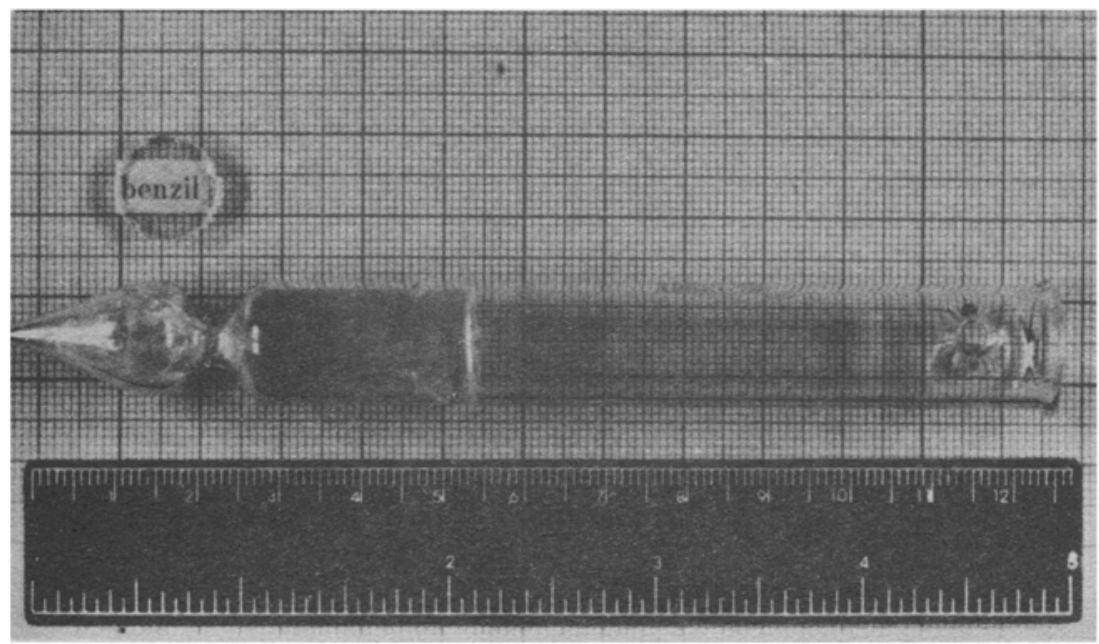

Figure 3. Photograph of as-grown (along with the ampoule) and cut and polished benzil single crystal.

$(100 \mathrm{kHz}-10 \mathrm{MHz})$, using the impedance/gain-phase analyser (HP 4194A) in conjunction with an indigenously built temperature variation cell. An ac-field of $\approx 6.7 \mathrm{~V} / \mathrm{cm}$ was applied across the crystal for the dielectric and impedance measurements. The measurements were performed, while both heating and cooling, with $\approx 15 \mathrm{~min}$ dwelling time at each temperature. The sample was mounted in such a way that it could vibrate freely. The piezoelectrically induced resonant vibrational frequencies were used for the determination of the stiffness coefficient $\left(C_{11}\right)$.

\section{Results and discussion}

A typical temperature profile of the BS furnace, used for the growth of benzil single crystal is shown in figure 2. Interestingly, it was found that one need not adopt a sealed ampoule technique for growing single crystals of benzil. A typical crystal growth run, including the slow cooling process, took about 9 days. The photograph of single crystals of benzil grown by Bridgman-Stockbarger technique, is depicted in figure 3 .

The dielectric constant $\left(\varepsilon_{\mathrm{r}}\right)$ and the loss tangent $(\tan \delta)$, measured along the crystallographic $a$-axis, as a function of frequency $(100 \mathrm{kHz}-10 \mathrm{MHz})$ are shown in figures $4 \mathrm{a}$ and $b$, respectively. One would notice here the presence of sharp resonance peaks in the frequency range $100-300 \mathrm{kHz}$. Measurements made on single crystal samples of varied dimensions indicate a strong dependence of the resonance frequencies on the size and shape of the sample. The benzil crystal, being piezoelectric, has numerous vibrational frequencies depending on its dimensions. The origin of the appearance of these peaks is attributed to the piezoelectric vibrations of the crystal at one of its resonant frequencies, stimulated by the external field applied across its thickness (i.e. along $a$-axis). Standing waves are established in the crystal due to the inverse piezoelectric effect and at specific frequencies of the applied field, resonance effects are expected. Coupling may exist between various possible vibrational modes, because of the complexity of the elastic 

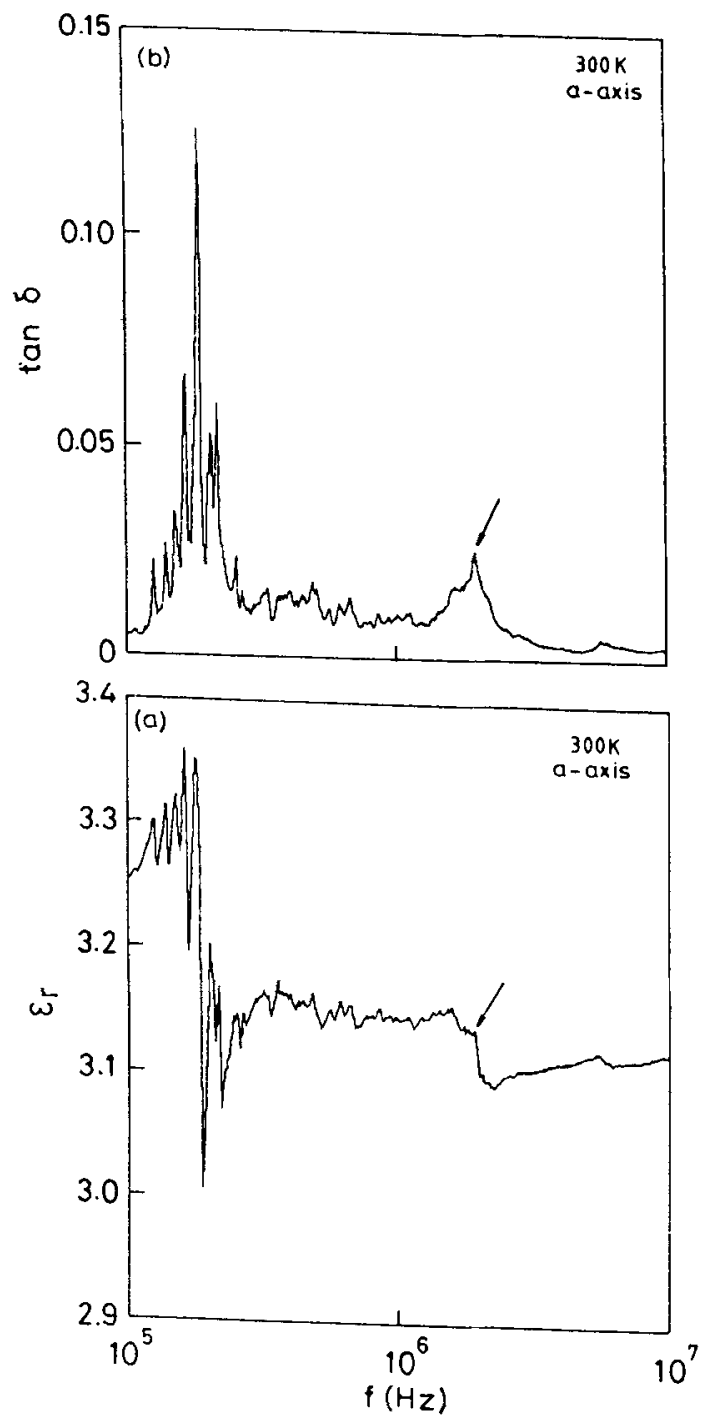

Figure 4. Frequency response of (a) $\varepsilon_{\mathrm{r}}$ and (b) $\tan \delta$ of benzil single crystal, monitored along the a-axis.

conditions. This leads to the simultaneous existence of many resonant frequencies comprised within a narrow frequency band (Mason 1950; Cady 1964). Therefore, the present findings are attributed to the piezoelectric nature of the crystal (Shankar and Varma 1996). Figure 5 shows variation of the dielectric constant and the loss tangent $(\tan \delta)$ as a function of frequency in the region of interest $(100-300 \mathrm{kHz})$. The above observation is corroborated by the existence of resonance peaks (though much smaller in magnitude) in the frequency response of the impedance $(Z)$ of the sample, around the same frequencies (figure 6).

The fall in $\varepsilon_{\mathrm{r}}$ in the $1-3 \mathrm{MHz}$ region (indicated by an arrow in figure $4 \mathrm{a}$ ) accompanied by a simultaneous rise in $\tan \delta$ (indicated by an arrow in figure $4 \mathrm{~b}$ ), is attributable to 

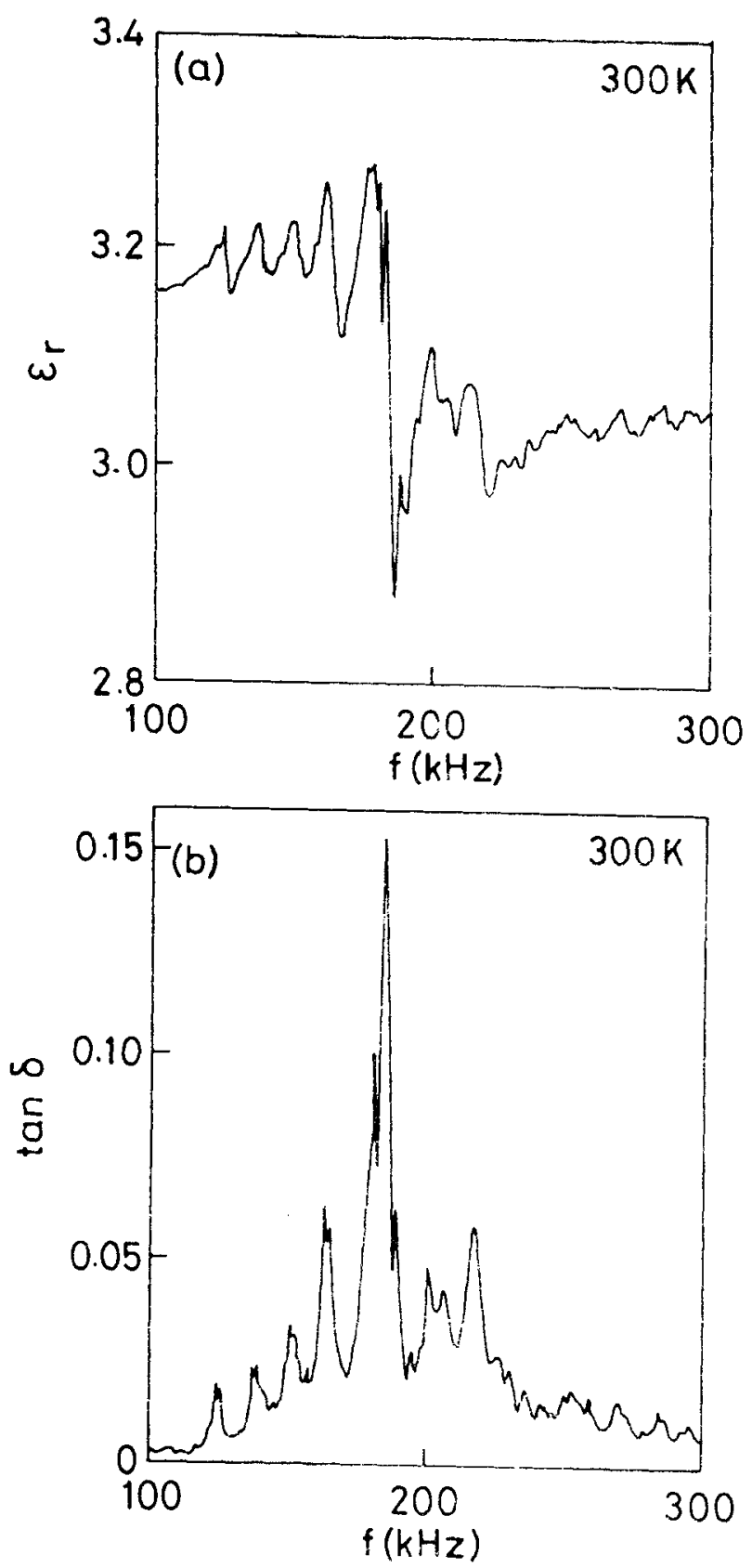

Figure 5. The variation of $(\mathbf{a}) \varepsilon_{\mathrm{r}}$ and $(\mathbf{b})$ ian $\delta$, with frequency, in the $100.300 \mathrm{lHz}$ region.

the relaxation of the dielectric absorption of the sample under study. On the other hand, the time-dependent preferential reorientation of the defects between two crystallographically equivalent orientations, $a$ and $b$-axes in the present case (figure 7). on the application of an ac-field along one of these directions can give rise to relaxation 


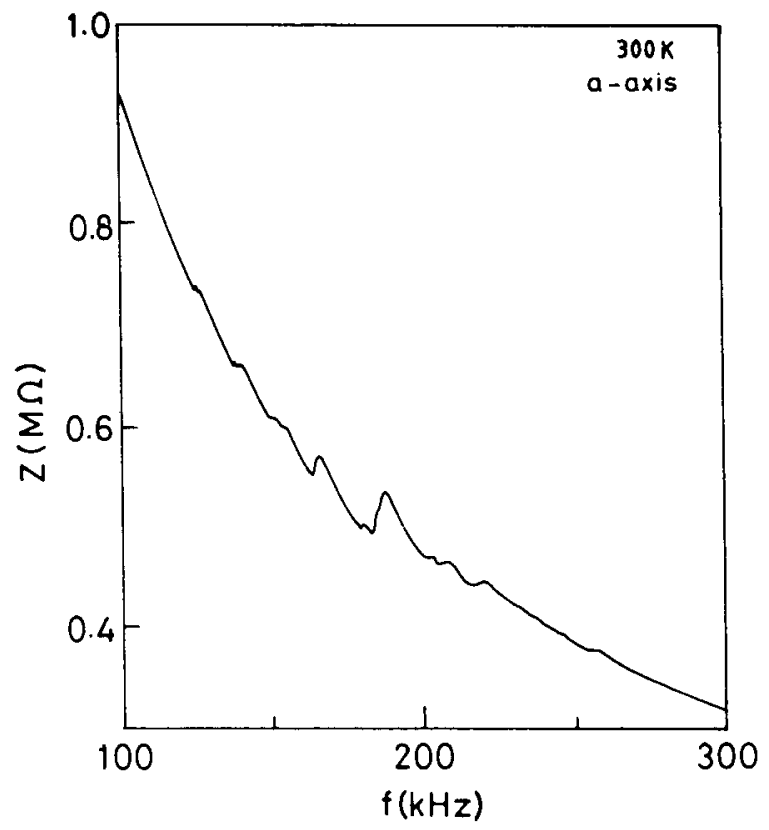

Figure 6. The variation of the impedance $(Z)$ with frequency.

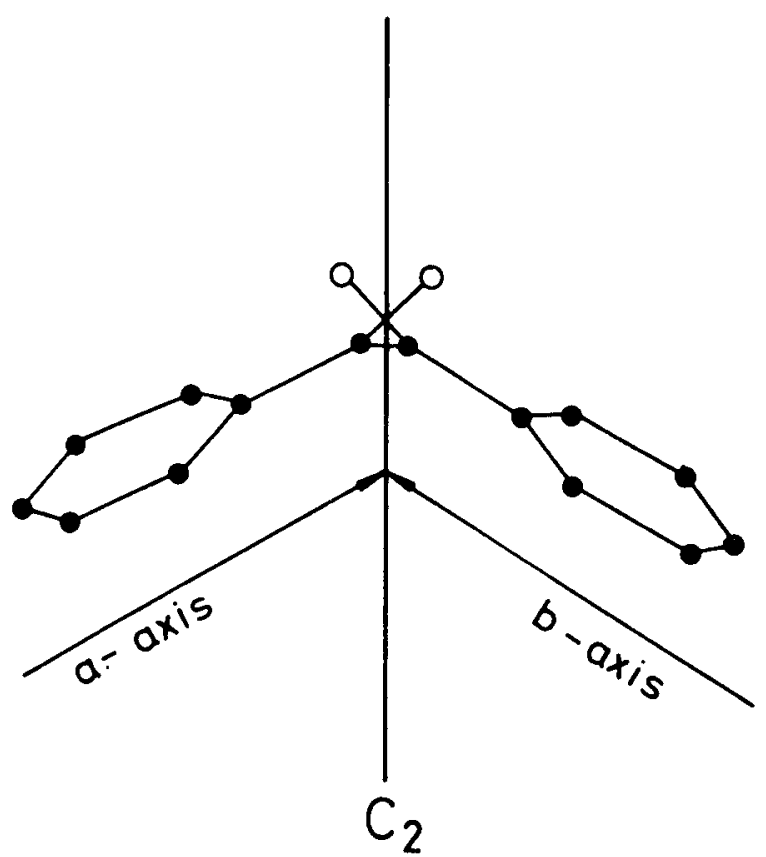

Figure 7. Benzil molecule in projection on (0001).

processes of the kind observed here. This argument is supported by the fact that the variation of $\varepsilon_{\mathrm{r}}$ with frequency, monitored along the c-axis, did not exhibit a relaxation of observable magnitude (Shankar et al 1994). 


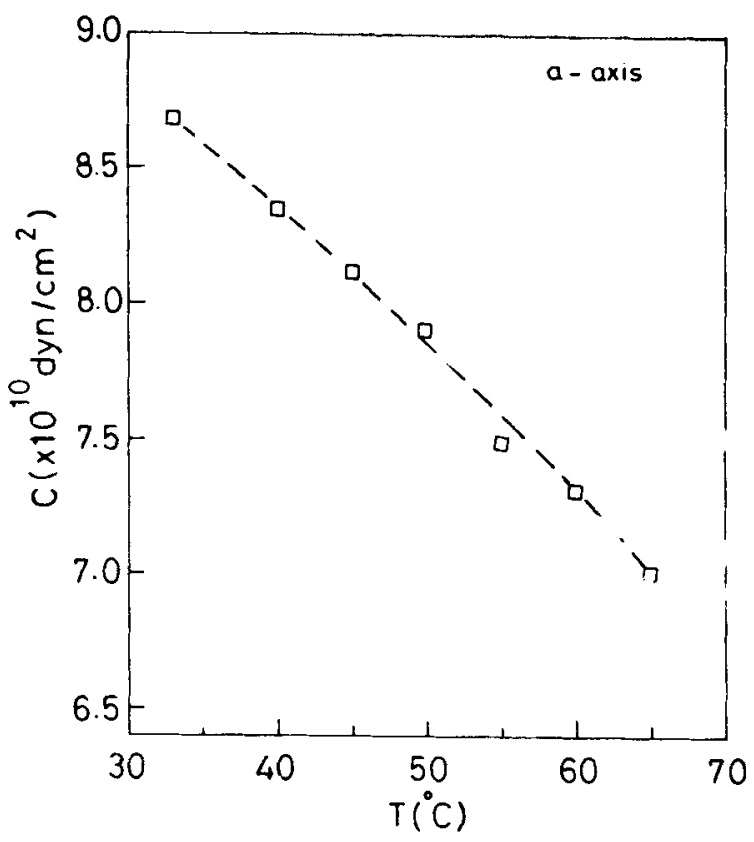

Figure 8. The variation of the stiffness coefficient $\left(C_{11}\right)$, of benzil single crystal, with temperature.

Investigations into the temperature dependence of the positions of the resonance peaks indicate a remarkable shift towards lower frequencies with increasing temperature. The stiffness coefficient $\left(C_{11}\right)$, which is the reciprocal of the elastic coefficient, evaluated based on the resonance--anti-resonance method is found to be comparable with the value measured by the Schaefer-Bergman method (diffraction of light by ultrasonic waves) at $300 \mathrm{~K}$ (Haussühl 1967). It is found to decrease steadily with increase in temperature (figure 8). Since the elastic compliance is supposed to exhibit a maximum around the melting point. the stiffness coefficient is expected to be minimum. Benzil does not exhibit any phase transition, above room temperature, before reaching its melting point. Hence no anomalies are expected in $C_{11}$, unlike in the case of commonly known piezoelectric material quartz, in the range of temperatures under study. It should be noted that $C_{11}$ is related to the longitudinal elastic mode propagating along the two-fold axis of the crystal. Since the frequency of natural oscillation is given by $(1 / 2 \pi) \sqrt{C / m}$ ( $m$ being the mass of the sample), a decrease in $C$, is expected to lead to a lowering of the vibration frequency. Hence the shift in the positions of the peaks towards lower frequencies with increase in temperature may be attributed to the decrease in the stiffness coefficient of the crystal.

\section{Conclusions}

Dielectric dispersion, caused by a piezoelectric resonance, is observed in the $100-$ $300 \mathrm{kHz}$ range. The resonance, the position of which has been found to be dependent on the dimensions of the sample under study, is attributed to its piezoelectric nature. The shift in the positions of the resonance peaks towards lower frequencies, with increase in 
temperature, is attributed to the decrease in the stiffness coefficient $\left(C_{11}\right)$ of the crystal. Since our prime interest is on the characterization of the resonance-enhanced electrooptic response of organic crystals. emphasis has been made on the dielectric dispersion in the $100-300 \mathrm{kHz}$ region of the frequency spectrum. Studies are in progress to visualize the influence of resonance effects on the electro-optic properties of these crystals.

\section{References}

Bosshard Ch, Sutter K, Schlesser R and Günter P 1993 J. Opt. Soc. Am. B10 867

Brown C J and Sadanaga R 1965 Acta Crystallogr. 18158

Cady W G 1964 Piezoelectricity (New York: Dover Press)

Haussühl V S 1967 Acta Crystallogr. A23 666

Jerphagnon J 1971 IEEE J. Quantum Electronics QE-7 42

Mason W P 1950 Piezoelectric crystals and their application to ultrasonics (New York: D. Van Nostrand)

Shankar M V and Varma K B R 1996 Ferroelectrics Lett. 2155

Shankar M V. Varma K B R. Reddy T J and Srikrishna A 1994 SPIE Proceedings (New Delhi: Tata McGraw Hill) p. 63 